\title{
$p$-Norm SDD tensors and eigenvalue localization
}

Qilong Liu and Yaotang Li*

\author{
"Correspondence: \\ liyaotang@ynu.edu.cn \\ School of Mathematics and \\ Statistics, Yunnan University, \\ Kunming, Yunnan 650091, P.R. China
}

\begin{abstract}
We present a new class of nonsingular tensors ( $p$-norm strictly diagonally dominant tensors), which is a subclass of strong $\mathcal{H}$-tensors. As applications of the results, we give a new eigenvalue inclusion set, which is tighter than those provided by Li et al. (Linear Multilinear Algebra 64:727-736, 2016) in some case. Based on this set, we give a checkable sufficient condition for the positive (semi)definiteness of an even-order symmetric tensor.
\end{abstract}

Keywords: $p$-norm SDD tensor; strong $\mathcal{H}$-tensor; positive (semi)definiteness; eigenvalue localization

\section{Introduction}

Let $\mathbb{C}(\mathbb{R})$ denote the set of all complex (real) numbers, and $[n]:=\{1,2, \ldots, n\}$. An $m$ th-order $n$-dimensional complex (real) tensor, denoted by $\mathcal{A} \in \mathbb{C}^{[m, n]}\left(\mathbb{R}^{[m, n]}\right)$, is a multidimensional array of $n^{m}$ elements of the form

$$
\mathcal{A}=\left(a_{i_{1} i_{2} \cdots i_{m}}\right), \quad a_{i_{1} \cdots i_{m}} \in \mathbb{C}(\mathbb{R}), i_{j} \in[n], j \in[m] .
$$

When $m=2, \mathcal{A}$ is an $n$-by- $n$ matrix. A tensor $\mathcal{A}=\left(a_{i_{1} \cdots i_{m}}\right) \in \mathbb{R}^{[m, n]}$ is called nonnegative if each its entry is nonnegative, and it is called symmetric $[2,3]$ if

$$
a_{i_{1} \cdots i_{m}}=a_{\pi\left(i_{1} \cdots i_{m}\right)}, \quad \forall \pi \in \Pi_{m},
$$

where $\Pi_{m}$ is the permutation group of $m$ indices. Moreover, an $m$ th-order $n$-dimensional tensor $\mathcal{I}=\left(\delta_{i_{1} i_{2} \cdots i_{m}}\right)$ is called the identity tensor [4] if

$$
\delta_{i_{1} i_{2} \cdots i_{m}}= \begin{cases}1 & \text { if } i_{1}=i_{2}=\cdots=i_{m} \\ 0 & \text { otherwise }\end{cases}
$$

For an $n$-dimensional vector $x=\left(x_{1}, x_{2}, \ldots, x_{n}\right)^{T}$, real or complex, we define the $n$-dimensional vector

$$
\mathcal{A} x^{m-1}:=\left(\sum_{i_{2}, \ldots, i_{m} \in[n]} a_{i i_{2} \cdots i_{m}} x_{i_{2}} \cdots x_{i_{m}}\right)_{1 \leq i \leq n},
$$

(C) 2016 Liu and Li. This article is distributed under the terms of the Creative Commons Attribution 4.0 International License (http://creativecommons.org/licenses/by/4.0/), which permits unrestricted use, distribution, and reproduction in any medium, provided you give appropriate credit to the original author(s) and the source, provide a link to the Creative Commons license, and indicate if changes were made. 
and the $n$-dimensional vector

$$
x^{[m-1]}:=\left(x_{i}^{m-1}\right)_{1 \leq i \leq n} .
$$

The following definition related to eigenvalues of tensors was first introduced and studied by Qi [3] and Lim [5].

Definition $1[3,5]$ Let $\mathcal{A}=\left(a_{i_{1} i_{2} \ldots i_{m}}\right) \in \mathbb{C}^{[m, n]}$. A pair $(\lambda, x) \in \mathbb{C} \times\left(\mathbb{C}^{n} \backslash\{0\}\right)$ is called an eigenvalue-eigenvector (or simply eigenpair) of $\mathcal{A}$ if satisfies the equation

$$
\mathcal{A} x^{m-1}=\lambda x^{[m-1]} .
$$

We call $(\lambda, x)$ an H-eigenpair if they are both real.

In addition, the spectral radius of a tensor $\mathcal{A}$ is defined as

$$
\rho(\mathcal{A})=\max \{|\lambda|: \lambda \text { is an eigenvalue of } \mathcal{A}\} .
$$

Definition 2 A tensor $\mathcal{A} \in \mathbb{C}^{[m, n]}$ is said to be nonsingular if zero is not an eigenvalue of $\mathcal{A}$. Otherwise, it is called singular.

Tensor eigenvalue problems have gained special attention in the realm of numerical multilinear algebra, and they have a wide range in practice; see [3, 4, 6-16]. For instance, we can use the smallest $\mathrm{H}$-eigenvalues of tensors to determine their positive (semi)definiteness, that is, for an even-order real symmetric tensor $\mathcal{A}$, if its smallest $\mathrm{H}$ eigenvalue is positive (nonnegative), then $\mathcal{A}$ is positive (semi)definite; consequently, the multivariate homogeneous polynomial $f(x)$ determined by $\mathcal{A}$ is positive (semi)definite [3].

Most often, it is difficult to compute the smallest $\mathrm{H}$-eigenvalue. Therefore, we always try to give a distribution range of eigenvalues of a given tensor in the complex plane. In particular, if this range is in the right-half complex plane, which means that the smallest $\mathrm{H}$-eigenvalue is positive, then the corresponding tensor is positive definite.

Qi [3] generalized the Geršgorin eigenvalue inclusion theorem from matrices to real symmetric tensors, which can be easily extended to generic tensors; see $[4,17]$.

Theorem 1 Let $\mathcal{A}=\left(a_{i_{1} i_{2} \cdots i_{m}}\right) \in \mathbb{C}^{[m, n]}$. Then

$$
\sigma(\mathcal{A}) \subseteq \Gamma(\mathcal{A})=\bigcup_{i \in[n]} \Gamma_{i}(\mathcal{A})
$$

where $\sigma(\mathcal{A})$ is the set of all the eigenvalues of $\mathcal{A}$, and

$$
\Gamma_{i}(\mathcal{A})=\left\{z \in \mathbb{C}:\left|z-a_{i i \cdots i}\right| \leq r_{i}(\mathcal{A})\right\}, \quad r_{i}(\mathcal{A})=\sum_{\substack{i_{2}, \ldots, i_{m} \in[n], \delta_{i i_{2} \cdots i_{m}}=0}}\left|a_{i i_{2} \cdots i_{m}}\right|
$$

Recently, as an extension of the theory in [18], Li et al. [1, 17, 19] proposed three new Brauer-type eigenvalue localization sets for tensors and showed tighter bounds than $\Gamma(\mathcal{A})$ 
of Theorem 1. We list the latest Brauer-type eigenvalue localization set as follows. For convenience, we denote

$$
\begin{aligned}
& \Delta_{i}=\left\{\left(i_{2}, i_{3}, \ldots, i_{m}\right): i_{j}=i \text { for some } j \in\{2,3, \ldots, m\}, \text { where } i, i_{2}, \ldots, i_{m} \in[n]\right\}, \\
& \bar{\Delta}_{i}=\left\{\left(i_{2}, i_{3}, \ldots, i_{m}\right): i_{j} \neq i \text { for any } j \in\{2,3, \ldots, m\}, \text { where } i, i_{2}, \ldots, i_{m} \in[n]\right\},
\end{aligned}
$$

and

$$
r_{i}^{\Delta_{i}}(\mathcal{A})=\sum_{\substack{\left(i_{2}, \ldots, i_{m}\right) \in \Delta_{i}, \delta_{i i_{2} \cdots i_{m}}=0}}\left|a_{i i_{2} \cdots i_{m}}\right|, \quad r_{i}^{\overline{\Delta_{i}}}(\mathcal{A})=\sum_{\left(i_{2}, \ldots, i_{m}\right) \in \bar{\Delta}_{i}}\left|a_{i i_{2} \cdots i_{m}}\right| .
$$

Theorem 2 [1] Let $\mathcal{A}=\left(a_{i_{1} i_{2} \cdots i_{m}}\right) \in \mathbb{C}^{[m, n]}$. Then

$$
\sigma(\mathcal{A}) \subseteq \Omega(\mathcal{A})=\left(\bigcup_{i \in[n]} \hat{\Omega}_{i}(\mathcal{A})\right) \cup\left(\bigcup_{\substack{i, j \in[n], i \neq j}}\left(\hat{\Omega}_{i, j}(\mathcal{A}) \cap \Gamma_{i}(\mathcal{A})\right)\right)
$$

where

$$
\hat{\Omega}_{i}(\mathcal{A})=\left\{z \in \mathbb{C}:\left|z-a_{i \cdots i}\right| \leq r_{i}^{\Delta_{i}}(\mathcal{A})\right\}
$$

and

$$
\hat{\Omega}_{i, j}(\mathcal{A})=\left\{z \in \mathbb{C}:\left(\left|z-a_{i \cdots i}\right|-r_{i}^{\Delta_{i}}(\mathcal{A})\right)\left(\left|z-a_{j \cdots j}\right|-r_{j}^{\bar{\Delta}_{i}}(\mathcal{A})\right) \leq r_{i}^{\bar{\Delta}_{i}}(\mathcal{A}) r_{j}^{\Delta_{i}}(\mathcal{A})\right\} .
$$

Li et al. [1] proved that the set $\Omega(\mathcal{A})$ in Theorem 2 is tighter than $\Theta(\mathcal{A})$ in [19] and $\mathcal{K}(\mathcal{A})$ in [17]; for details, see Theorem 2.3 in [1].

In this paper, we continue this research on the eigenvalue localization problem for tensors. A class of strictly diagonally dominant tensors that involve a parameter $p$ in the interval $[1, \infty]$, denoted by $p$-norm SDD tensor, is introduced in Section 2. In Section 3, we discuss the relationships between $p$-norm SDD tensors and strong $\mathcal{H}$-tensors. A new eigenvalue inclusion set for tensors based on $p$-norm SDD tensors is obtained in Section 4 , and numerical results show that the new set is tighter than $\Omega(\mathcal{A})$ in Theorem 2 in some case. Finally, in Section 5, we give a checkable sufficient condition for the positive (semi)definiteness of even-order symmetric tensors.

\section{$2 p$-Norm SDD tensors}

In this section, we propose a new class of nonsingular tensors, namely $p$-norm strictly diagonally dominant tensors. First, some notation and the definition of strictly diagonally dominant tensors are given.

Given a tensor $\mathcal{A}=\left(a_{i_{1} i_{2} \cdots i_{m}}\right) \in \mathbb{C}^{[m, n]}$ and a real number $p \in[1, \infty]$, denote

$$
r_{i}^{p}(\mathcal{A}):=\left(\sum_{\substack{i_{2}, \ldots, i_{m} \in[n], \delta_{i i_{2} \cdots i_{m}=0}}}\left|a_{i i_{2} \cdots i_{m}}\right|^{p}\right)^{\frac{1}{p}} \quad \text { for all } i \in[n]
$$


In particular, if $p=1$, then $r_{i}^{1}(\mathcal{A})=r_{i}(\mathcal{A})$ for all $i \in[n]$. If $p=\infty$, then $r_{i}^{\infty}(\mathcal{A})=$ $\max _{\substack{i_{2}, \ldots, i_{m} \in[n] \\ \delta i i_{2} \cdots i_{m}=0}}\left|a_{i i_{2} \cdots i_{m}}\right|$ for all $i \in[n]$. For a vector $x=\left(x_{1}, x_{2}, \ldots, x_{n}\right)^{T} \in \mathbb{C}^{n}$, the $l_{q}$-norm on $\mathbb{C}^{n}$ is

$$
\|x\|_{q}:=\left(\sum_{i \in[n]}\left|x_{i}\right|^{q}\right)^{\frac{1}{q}} .
$$

Definition 3 [16] A tensor $\mathcal{A}=\left(a_{i_{1} i_{2} \cdots i_{m}}\right) \in \mathbb{C}^{[m, n]}$ is diagonally dominant if

$$
\left|a_{i i \cdots i}\right| \geq r_{i}(\mathcal{A}) \text { for all } i \in[n] \text {, }
$$

and $\mathcal{A}$ is strictly diagonally dominant if the strict inequality holds in (2) for all $i$.

$\operatorname{Remark} \mathbf{1} \mathcal{A}=\left(a_{i_{1} i_{2} \cdots i_{m}}\right) \in \mathbb{C}^{[m, n]}$ is strictly diagonally dominant if and only if

$$
\max _{i \in[n]} \frac{r_{i}(\mathcal{A})}{\left|a_{i i \cdots i}\right|}<1 .
$$

It is well known that strictly diagonally dominant tensors are nonsingular. An interesting problem arises: for a tensor $\mathcal{A}=\left(a_{i_{1} i_{2} \cdots i_{m}}\right) \in \mathbb{C}^{[m, n]}$ satisfying

$$
\max _{i \in[n]} \frac{r_{i}^{p}(\mathcal{A})}{\left|a_{i i \cdots i}\right|}<1,
$$

is $\mathcal{A}$ nonsingular or not? Certainly, when $p=1, \mathcal{A}$ is a strictly diagonally dominant tensor, which means that $\mathcal{A}$ is nonsingular, but when $p>1, \mathcal{A}$ may be singular as the following simple example shows.

Example 1 Let $\mathcal{A}=\left(a_{i j k}\right) \in \mathbb{R}^{[3,2]}$, where

$$
a_{111}=a_{222}=-3, \quad \text { and the remaining } \quad a_{i j k}=1 \text {. }
$$

Then, since $\mathcal{A} e^{2}=0$, where $e=(1,1,1)^{T}$, this implies $0 \in \sigma(\mathcal{A})$. However, for every $p>1$, we have

$$
\max _{i \in[2]} \frac{r_{i}^{p}(\mathcal{A})}{\left|a_{i i i}\right|}=3^{\frac{1-p}{p}}<1 .
$$

Therefore, something needs to be added in order to obtain a nonsingular $\mathcal{A}$ for a real number $p \in(1, \infty]$. We provide an answer further, but we first introduce a class of strictly diagonally dominant tensors that involve a parameter $p$ in the interval $[1, \infty]$.

Definition 4 Let $\mathcal{A}=\left(a_{i_{1} i_{2} \cdots i_{m}}\right) \in \mathbb{C}^{[m, n]}$ and $p \in[1, \infty], \mathcal{A}$ is called a $p$-norm strictly diagonally dominant tensor (or, shortly, $p$-norm SDD tensor) if

$$
\left\|\delta_{p}(\mathcal{A})\right\|_{q}<1
$$


where

$$
\delta_{p}(\mathcal{A}):=\left(\delta_{1}, \delta_{2}, \ldots, \delta_{n}\right)^{T}, \quad \delta_{i}:=\left(\frac{r_{i}^{p}(\mathcal{A})}{\left|a_{i \cdots i}\right|}\right)^{\frac{1}{m-1}} \text { for all } i \in[n],
$$

and $q$ is Hölder's complement of $p$, that is, $\frac{1}{p}+\frac{1}{q}=1$.

Remark 2 Definition 4 extends the concept of $\operatorname{SDD}(p)$ matrix given in [20] to tensors. Clearly, the $\operatorname{SDD}(p)$ matrix is a 2nd-order $p$-norm SDD tensor.

Remark 3 Taking $p=1, \mathcal{A}$ is a 1 -norm SDD tensor if and only if

$$
\left\|\delta_{1}(\mathcal{A})\right\|_{\infty}=\max _{i \in[n]}\left(\frac{r_{i}(\mathcal{A})}{\left|a_{i i \cdots i}\right|}\right)^{\frac{1}{m-1}}<1,
$$

that is,

$$
\max _{i \in[n]} \frac{r_{i}(\mathcal{A})}{\left|a_{i i \cdots i}\right|}<1
$$

which is equivalent to the fact that $\mathcal{A}$ is a strictly diagonally dominant tensor. The other extreme case is $p=\infty$. $\mathcal{A}$ is a $\infty$-norm SDD tensor if and only if

$$
\left\|\delta_{\infty}(\mathcal{A})\right\|_{1}=\sum_{i \in[n]}\left(\frac{\max _{i_{2}, \ldots, i_{m} \in[n]},\left|a_{i i_{2} \cdots i_{m}}\right|}{\delta_{i i_{2} \cdots i_{m}}=0}\right)^{\frac{1}{m-1}}<1 .
$$

The $p$-norm SDD tensors can also be characterized in the following way.

Proposition 1 Let $\mathcal{A} \in \mathbb{C}^{[m, n]}$ and $p \in[1, \infty]$. Then $\mathcal{A}$ is a $p$-norm $S D D$ tensor if and only if there exists an entrywise positive vector $x=\left(x_{1}, x_{2}, \ldots, x_{n}\right)^{T} \in \mathbb{R}^{n}$ such that $\|x\|_{q} \leq 1$, where $q$ is Hölder's complement of $p$ such that

$$
x_{i}^{m-1}\left|a_{i \cdots i}\right|>r_{i}^{p}(\mathcal{A}) \quad \text { for all } i \in[n] .
$$

Proof Necessity. Suppose that $\mathcal{A}$ is a $p$-norm SDD tensor. It follows from inequality (3) of Definition 4 that there exists a sufficiently small $\varepsilon>0$ such that, for $x_{i}:=\delta_{i}+\varepsilon>0$, where $i \in[n],\|x\|_{q} \leq 1$. Thus, $x_{i}^{m-1}>\delta_{i}^{m-1}=\frac{r_{i}^{p}(\mathcal{A})}{\left|a_{i \cdots i}\right|}$, which implies inequality (4).

Sufficiency. Suppose that there exists an entrywise positive vector $x>0$ such that $\|x\|_{q} \leq$ 1 and inequality (4) holds. By inequality (4) we have

$$
x_{i}^{m-1}>\frac{r_{i}^{p}(\mathcal{A})}{\left|a_{i \cdots i}\right|}=\delta_{i}^{m-1} \quad \text { for all } i \in[n],
$$

which implies $x_{i}>\delta_{i}$ for all $i \in[n]$, which, together with $\|x\|_{q} \leq 1$, yields

$$
\left\|\delta_{p}(\mathcal{A})\right\|_{q}<\|x\|_{q} \leq 1 .
$$

Thus, $\mathcal{A}$ is a $p$-norm SDD tensor. The proof is completed.

The following result proves the nonsingular of $p$-norm SDD tensors. 
Theorem 3 Let $\mathcal{A} \in \mathbb{C}^{[m, n]}$ be a p-norm SDD tensor. Then $\mathcal{A}$ is nonsingular.

Proof Suppose that $\mathcal{A}$ is singular, that is, $0 \in \sigma(\mathcal{A})$. It follows from equality (1) that there exists $y \in \mathbb{C}^{n} \backslash\{0\}$, such that

$$
\mathcal{A} y^{m-1}=0 \text {. }
$$

Without the loss of generality, we can assume that $\|y\|_{q}=1$. Then, equality (5) yields

$$
a_{i \cdots i} y_{i}^{m-1}=-\sum_{\substack{i_{2}, \ldots, i_{m} \in[n], \delta_{i i_{2} \cdots i_{m}=0}}} a_{i i_{2} \cdots i_{m}} y_{i_{2}} \cdots y_{i_{m}} \quad \text { for all } i \in[n] \text {, }
$$

which implies that

$$
\left|a_{i \cdots i}\right|\left|y_{i}\right|^{m-1}=\left|\sum_{\substack{i_{2}, \ldots, i_{m} \in[n], \delta_{i i_{2} \cdots i_{m}=0}=0}} a_{i i_{2} \cdots i_{m}} y_{i_{2}} \cdots y_{i_{m}}\right| \quad \text { for all } i \in[n] .
$$

Then, applying the Hölder inequality to the right-hand side of equality (6), we obtain

$$
\begin{aligned}
\left|a_{i \cdots i}\right|\left|y_{i}\right|^{m-1} & \leq\left(\sum_{\substack{i_{2}, \ldots, i_{m} \in[n], \delta_{i i_{2} \cdots i_{m}}=0}}\left|a_{i i_{2} \cdots i_{m}}\right|^{p}\right)^{\frac{1}{p}}\left(\sum_{\substack{i_{2}, \ldots, i_{m} \in[n], \delta_{i_{2} \cdots i_{m}}=0}}\left|y_{i_{2}} \cdots y_{i_{m}}\right|^{q}\right)^{\frac{1}{q}} \\
& =r_{i}^{p}(\mathcal{A})\left[\left(\sum_{j=1}^{n}\left|y_{j}\right|^{q}\right)^{m-1}-\left|y_{i}\right|^{(m-1) q}\right]^{\frac{1}{q}} \\
& =r_{i}^{p}(\mathcal{A})\left(\|y\|_{q}^{(m-1) q}-\left|y_{i}\right|^{(m-1) q}\right)^{\frac{1}{q}} \\
& \leq r_{i}^{p}(\mathcal{A})\|y\|_{q}^{m-1} \\
& =r_{i}^{p}(\mathcal{A}) \quad \text { for all } i \in[n] .
\end{aligned}
$$

Since $\mathcal{A}$ is a $p$-norm SDD tenor, there exists an entrywise positive vector $x>0$ such that $\|x\|_{q} \leq 1$ and inequality (4) holds. Combining inequality (4) with (7), we obtain

$$
\left|a_{i \cdots i}\right|\left|y_{i}\right|^{m-1} \leq r_{i}^{p}(\mathcal{A})<x_{i}^{m-1}\left|a_{i \cdots i}\right| \quad \text { for all } i \in[n],
$$

which means that

$$
\left|y_{i}\right|<x_{i} \quad \text { for all } i \in[n] .
$$

Thus, $\|x\|_{q}>\|y\|_{q}=1$, which contradicts $\|x\|_{q} \leq 1$. The proof is completed.

\section{Relationships between $p$-norm SDD tensors and strong $\mathcal{H}$-tensors}

The following lemma shows that the strong $\mathcal{H}$-tensors play an important role in identifying the positive definiteness of even-order real symmetric tensors.

Lemma 1 [21] Let $\mathcal{A}=\left(a_{i_{1} \cdots i_{m}}\right) \in \mathbb{R}^{[m, n]}$ be an even-order real symmetric tensor with $a_{i \cdots i}>$ 0 for all $i \in[n]$. If $\mathcal{A}$ is a strong $\mathcal{H}$-tensor, then $\mathcal{A}$ is positive definite. 
It is known that the strictly diagonally dominant tensors are a subclass of strong $\mathcal{H}$-tensors. An interesting problem arises: whether the class of $p$-norm SDD tensors is a subclass of strong $\mathcal{H}$-tensors for an arbitrary $p \in[1, \infty]$. In this section, we discuss this problem. We first recall the definition of strong $\mathcal{H}$-tensors.

Definition 5 [16] A tenor $\mathcal{A}=\left(a_{i_{1} i_{2} \cdots i_{m}}\right) \in \mathbb{R}^{[m, n]}$ is called an $\mathcal{M}$-tensor if there exist a nonnegative tensor $\mathcal{B}$ and a positive real number $\eta \geq \rho(\mathcal{B})$ such that $\mathcal{A}=\eta \mathcal{I}-\mathcal{B}$. If $\eta>\rho(\mathcal{B})$, then $\mathcal{A}$ is called a strong $\mathcal{M}$-tensor.

Definition 6 [9] Let $\mathcal{A}=\left(a_{i_{1} i_{2} \cdots i_{m}}\right) \in \mathbb{C}^{[m, n]}$. We call another tensor $\mathcal{M}(\mathcal{A})=\left(m_{i_{1} i_{2} \cdots i_{m}}\right)$ the comparison tensor of $\mathcal{A}$ if

$$
m_{i_{1} i_{2} \cdots i_{m}}= \begin{cases}+\left|a_{i_{1} i_{2} \cdots i_{m}}\right| & \text { if }\left(i_{2}, i_{3}, \ldots, i_{m}\right)=\left(i_{1}, i_{1}, \ldots, i_{1}\right), \\ -\left|a_{i_{1} i_{2} \cdots i_{m}}\right| & \text { if }\left(i_{2}, i_{3}, \ldots, i_{m}\right) \neq\left(i_{1}, i_{1}, \ldots, i_{1}\right) .\end{cases}
$$

Definition 7 [9] We call a tensor an $\mathcal{H}$-tensor if its comparison tensor is an $\mathcal{M}$-tensor. We call it a strong $\mathcal{H}$-tensor if its comparison tensor is a strong $\mathcal{M}$-tensor.

Note that Li et al. [21] also provided an equivalent definition of strong $\mathcal{H}$-tensors; for details, see [21].

In [22], the multiplication of matrices has been extended to tensors. In the following, we state these results for reference.

Definition 8 [22] Let $\mathcal{A}=\left(a_{i_{1} i_{2} \cdots i_{m}}\right)$ and $\mathcal{B}=\left(b_{i_{1} i_{2} \cdots i_{k}}\right)$ be $n$-dimensional tensors of orders $m \geq 2$ and $k \geq 1$, respectively. The product $\mathcal{A B}$ is the following $n$-dimensional tensor $\mathcal{C}$ of order $(m-1)(k-1)+1$ with entries

$$
c_{i \alpha_{1} \alpha_{2} \cdots \alpha_{m-1}}=\sum_{i_{2}, \ldots, i_{m} \in[n]} a_{i i_{2} \cdots i_{m}} b_{i_{2} \alpha_{1}} \cdots b_{i_{m} \alpha_{m-1}}
$$

where $i \in[n]$ and $\alpha_{1}, \ldots, \alpha_{m-1} \in\left\{j_{2} j_{3} \ldots j_{k}: j_{l} \in[n], l=2,3, \ldots, k\right\}$.

Remark 4 When $m=2$ and $\mathcal{A}=\left(a_{i j}\right)$ is a matrix of dimension $n$, then $\mathcal{A B}$ is an $m$ th-order $n$-dimensional tensor, and we have

$$
(\mathcal{A B})_{i_{1} i_{2} \cdots i_{m}}=\sum_{l_{2} \in[n]} a_{i_{1} l_{2}} b_{l_{2} i_{2} \cdots i_{m}}, \quad i_{j} \in[n], j \in[m] .
$$

In particular, the product of a diagonal matrix $X=\operatorname{diag}\left(x_{1}, x_{2}, \ldots, x_{n}\right)$ and the tensor $\mathcal{A}$ is given by

$$
(X \mathcal{A})_{i_{1} i_{2} \ldots i_{m}}=x_{i_{1}} a_{i_{1} i_{2} \ldots i_{m}}, \quad i_{j} \in[n], j \in[m] .
$$

Remark 5 Given an $n$-by- $n$ matrix $X$ and two $m$ th order $n$-dimensional tensors $\mathcal{A}$, $\mathcal{B}$, we have the right distributive law for tensors [22], that is,

$$
X \cdot \mathcal{A}+X \cdot \mathcal{B}=X \cdot(\mathcal{A}+\mathcal{B})
$$


Based on this multiplication of tensors, Kannan, Shaked-Monderer, and Berman [23] established a necessary and sufficient condition for a tensor to be a strong $\mathcal{H}$-tensor.

Lemma 2 [23] Let $\mathcal{A} \in \mathbb{C}^{[m, n]}$. Then $\mathcal{A}$ is a strong $\mathcal{H}$-tensor if and only if $a_{i \cdots i} \neq 0$ for all $i \in[n]$ and

$$
\rho\left(\mathcal{I}-D_{\mathcal{M}(\mathcal{A})}^{-1} \mathcal{M}(\mathcal{A})\right)<1
$$

where $D_{\mathcal{M}(\mathcal{A})}$ is the diagonal matrix with the same diagonal entries as $\mathcal{M}(\mathcal{A})$.

The following lemma is given by Qi [3].

Lemma 3 [3] Let $\mathcal{A} \in \mathbb{C}^{[m, n]}$ and $\mathcal{B}=a(\mathcal{A}+b \mathcal{I})$, where $a$ and $b$ are two complex numbers. Then $\mu$ is an eigenvalue of $\mathcal{B}$ if and only if $\mu=a(\lambda+b)$ and $\lambda$ is an eigenvalue of $\mathcal{A}$. In this case, they have the same eigenvectors.

Next, we present an equivalence condition for singular tensors.

Lemma 4 Let $\mathcal{A} \in \mathbb{C}^{[m, n]}$. Then $\mathcal{A}$ is singular if and only if $D \mathcal{A}$ is singular, where $D=$ $\operatorname{diag}\left(d_{1}, d_{2}, \ldots, d_{n}\right)$ is a positive diagonal matrix.

Proof Suppose that $D \mathcal{A}$ is singular, that is, $0 \in \sigma(D \mathcal{A})$. Then there exists a vector $x=$ $\left(x_{1}, x_{2}, \ldots, x_{n}\right)^{T} \neq 0$ such that

$$
\sum_{i_{2}, \ldots, i_{m} \in[n]} d_{i} a_{i i_{2} \cdots i_{m}} x_{i_{2}} \cdots x_{i_{m}}=0 \quad \text { for all } i \in[n]
$$

which is equivalent to

$$
\sum_{i_{2}, \ldots, i_{m} \in[n]} a_{i i_{2} \cdots i_{m}} x_{i_{2}} \cdots x_{i_{m}}=0 \quad \text { for all } i \in[n]
$$

which implies $0 \in \sigma(\mathcal{A})$, that is, $\mathcal{A}$ is singular. The proof is completed.

By applying Lemmas 2, 3, and 4, we can now reveal the relationship of $p$-norm SDD tensors and strong $\mathcal{H}$-tensors.

Theorem 4 Let $\mathcal{A} \in \mathbb{C}^{[m, n]}$. If $\mathcal{A}$ is a p-norm SDD tensor, then $\mathcal{A}$ is a strong $\mathcal{H}$-tensor.

Proof By Lemma 2 the theorem will be proved if we can show that $\rho\left(\mathcal{I}-D_{\mathcal{M}(\mathcal{A})}^{-1} \mathcal{M}(\mathcal{A})\right)<1$. Assume, on the contrary, that there exists $\lambda \in \sigma\left(\mathcal{I}-D_{\mathcal{M}(\mathcal{A})}^{-1} \mathcal{M}(\mathcal{A})\right)$ such that $|\lambda| \geq 1$. Then, by Lemma 3 ,

$$
0 \in \sigma\left(\lambda \mathcal{I}-\mathcal{I}+D_{\mathcal{M}(\mathcal{A})}^{-1} \mathcal{M}(\mathcal{A})\right)
$$

According to the right distributive law for tensors, we have

$$
\lambda \mathcal{I}-\mathcal{I}+D_{\mathcal{M}(\mathcal{A})}^{-1} \mathcal{M}(\mathcal{A})=D_{\mathcal{M}(\mathcal{A})}^{-1}\left((\lambda-1) D_{\mathcal{M}(\mathcal{A})} \mathcal{I}+\mathcal{M}(\mathcal{A})\right)
$$


which, together with Lemma 4, yields

$$
0 \in \sigma\left((\lambda-1) D_{\mathcal{M}(\mathcal{A})} \mathcal{I}+\mathcal{M}(\mathcal{A})\right) .
$$

However, there exists an entrywise positive vector $x>0$ such that $\|x\|_{q} \leq 1$, and inequality (4) holds because $\mathcal{A}$ is a $p$-norm SDD tensor. By inequality (4) we have

$$
\begin{aligned}
x_{i}^{m-1}|(\lambda-1)| a_{i \cdots i}|+| a_{i \cdots i}|| & =x_{i}^{m-1}|\lambda|\left|a_{i \cdots i}\right| \\
& \geq x_{i}^{m-1}\left|a_{i \cdots i}\right| \\
& >r_{i}^{p}(\mathcal{A}) \\
& =r_{i}^{p}\left((\lambda-1) D_{\mathcal{M}(\mathcal{A})} \mathcal{I}+\mathcal{M}(\mathcal{A})\right),
\end{aligned}
$$

which implies that $(\lambda-1) D_{\mathcal{M}(\mathcal{A})} \mathcal{I}+\mathcal{M}(\mathcal{A})$ is a $p$-norm SDD tensor. By Theorem 3 we have

$$
0 \notin \sigma\left((\lambda-1) D_{\mathcal{M}(\mathcal{A})} \mathcal{I}+\mathcal{M}(\mathcal{A})\right)
$$

which contradicts (8). The proof is completed.

\section{Eigenvalue localization}

Similarly to matrices, a nonsingular class of tensors can lead to an eigenvalue localization result. In this section, we illustrate this fact with the class of $p$-norm SDD tensors.

Theorem 5 Let $\mathcal{A} \in \mathbb{C}^{[m, n]}$ and $p \in[1, \infty]$. Then

$$
\sigma(\mathcal{A}) \subseteq \Phi^{p}(\mathcal{A})
$$

When $p=1, \Phi^{1}(\mathcal{A})=\Gamma(\mathcal{A})$. When $p>1$,

$$
\Phi^{p}(\mathcal{A})=\left\{z \in \mathbb{C}: \sum_{i \in[n]}\left[\frac{r_{i}^{p}(\mathcal{A})}{\left|z-a_{i \cdots i}\right|}\right]^{\frac{p}{(m-1)(p-1)}} \geq 1\right\} .
$$

Proof Clearly, if $p=1, \sigma(\mathcal{A}) \subseteq \Gamma(\mathcal{A})$ can be easily obtained from Theorem 1 . If $p>1$, suppose that there exists $\lambda \in \sigma(\mathcal{A})$ such that $\lambda \notin \Phi^{p}(\mathcal{A})$, that is,

$$
\sum_{i \in[n]}\left[\frac{r_{i}^{p}(\mathcal{A})}{\left|\lambda-a_{i \cdots i}\right|}\right]^{\frac{p}{(m-1)(p-1)}}<1 .
$$

Let $\mathcal{B}:=\lambda \mathcal{I}-\mathcal{A}=\left(b_{i_{1} i_{2} \cdots i_{m}}\right)$. Since $\lambda \in \sigma(\mathcal{A})$, this, together with Lemma 3 , yields that $\mathcal{B}$ is surely singular. On the other hand, by the definition of $\mathcal{B}$ we obtain $r_{i}^{p}(\mathcal{B})=r_{i}^{p}(\mathcal{A})$ and $\left|b_{i \cdots i}\right|=\left|\lambda-a_{i \cdots i}\right|$ for all $i \in[n]$, so that (9) becomes

$$
\sum_{i \in[n]}\left[\frac{r_{i}^{p}(\mathcal{B})}{\left|b_{i \cdots i}\right|}\right]^{\frac{p}{(m-1)(p-1)}}<1
$$


which implies

$$
\left\|\delta_{p}(\mathcal{B})\right\|_{q}<1
$$

where $q$ is Hölder's complement of $p$, which means that $\mathcal{B}$ is a $p$-norm SDD tensor. By Theorem $3, \mathcal{B}$ is nonsingular. This leads to a contradiction.

Remark 6 When $m=2$, Theorem 5 reduces to the result of [20].

Remark 7 In particular, taking $p=\infty$, we have

$$
\Phi^{\infty}(\mathcal{A})=\left\{z \in \mathbb{C}: \sum_{i \in[n]}\left[\frac{\max _{i_{2, \ldots, i_{m} \in[n]},}\left|a_{i i_{2} \cdots i_{m}}\right|}{\delta_{i_{2} \cdots i_{m}=0} \mid}\right]^{\frac{1}{(m-1)}} \geq 1\right\} .
$$

It follows from Theorem 5 that

$$
\sigma(\mathcal{A}) \subseteq \Phi^{p}(\mathcal{A})
$$

but since this conclusion holds for any $p \in[1, \infty]$, we immediately have the following theorem.

Theorem 6 Let $\mathcal{A} \in \mathbb{C}^{[m, n]}$. Then

$$
\sigma(\mathcal{A}) \subseteq \bigcap_{p \in[1, \infty]} \Phi^{p}(\mathcal{A})
$$

where $\Phi^{p}(\mathcal{A})$ is defined as in Theorem 5.

The following example shows that $\Phi^{\infty}(\mathcal{A})$ is tighter than $\Omega(\mathcal{A})$ in some case.

Example 2 Let $\mathcal{A}=\left(a_{i j k}\right) \in \mathbb{R}^{[3,2]}$ and $\mathcal{B}=\left(b_{i j k}\right) \in \mathbb{R}^{[3,2]}$ with elements defined as follows:

$$
\begin{aligned}
& a_{111}=1.96, \quad a_{222}=16, \quad \text { and the remaining } \quad a_{i j k}=1, \\
& b_{111}=18, \quad b_{222}=16, \quad \text { and the remaining } \quad b_{i j k}=1,
\end{aligned}
$$

respectively. The eigenvalue inclusion regions $\Omega(\mathcal{A})(\Omega(\mathcal{B}))$, $\Phi^{\infty}(\mathcal{A})\left(\Phi^{\infty}(\mathcal{B})\right)$ and the exact eigenvalues of $\mathcal{A}(\mathcal{B})$ are drawn in Figure $1 \mathrm{~A}$ (Figure 1B), where $\Omega(\mathcal{A})\left(\Omega(\mathcal{B})\right.$ ), $\Phi^{\infty}(\mathcal{A})$ $\left(\Phi^{\infty}(\mathcal{B})\right)$ and the exact eigenvalues of $\mathcal{A}(\mathcal{B})$ are respectively denoted by the blue area, the green area, and red asterisks. In addition, by Corollary 7.8 in [10] we have

$$
\sigma(\mathcal{A})=\{15.6288,16.7844,1.7534+0.4687 i, 1.7534-0.4687 i\}
$$

and

$$
\sigma(\mathcal{B})=\{20.1811,17.3673,15.2258+0.5245 i, 15.2258-0.5245 i\} .
$$

It is easy to see that $\Phi^{\infty}(\mathcal{A}) \subseteq \Omega(\mathcal{A})$, but $\Omega(\mathcal{B}) \subseteq \Phi^{\infty}(\mathcal{B})$. 
Figure 1 The eigenvalue inclusion sets of tensors $\mathcal{A}$ and $\mathcal{B}$ for Example 2.
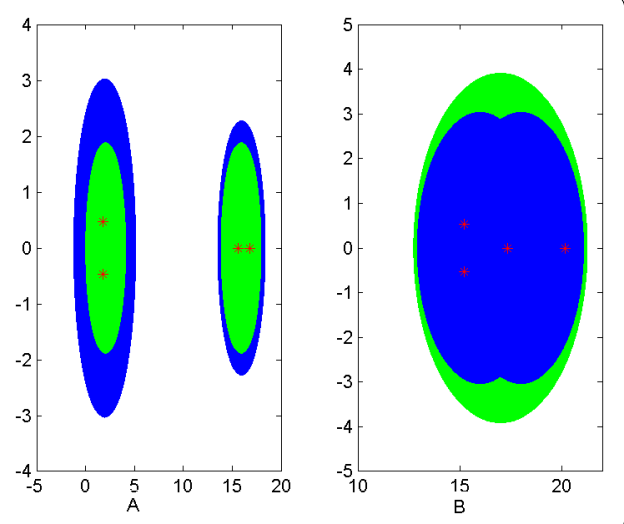

\section{Determining the positive (semi)definiteness for an even-order real symmetric tensor}

By applying the results obtained in Sections 3 and 4 we give a sufficient condition for the positive (semi)definiteness of an even-order real symmetric tensor.

Theorem 7 Let $\mathcal{A}=\left(a_{i_{1} i_{2} \ldots i_{m}}\right) \in \mathbb{R}^{[m, n]}$ be an even-order symmetric tensor with $a_{i \cdots i}>0$ for all $i \in[n]$. If $\mathcal{A}$ is a p-norm SDD tensor, then $\mathcal{A}$ is positive definite.

Proof The theorem follows immediately from Lemma 1 and Theorem 4.

Theorem 8 Let $\mathcal{A}=\left(a_{i_{1} i_{2} \ldots i_{m}}\right) \in \mathbb{R}^{[m, n]}$ be an even-order symmetric tensor with $a_{i \cdots i}>0$ for all $i \in[n]$, and $p \in[1, \infty]$. If

$$
\left\|\delta_{p}(\mathcal{A})\right\|_{q} \leq 1
$$

where $q$ is Hölder's complement of $p$. Then $\mathcal{A}$ is positive semidefinite.

Proof If $p=1$, then

$$
\left\|\delta_{1}(\mathcal{A})\right\|_{\infty}=\max _{i \in[n]}\left(\frac{r_{i}(\mathcal{A})}{\left|a_{i i \cdots i}\right|}\right)^{\frac{1}{m-1}} \leq 1,
$$

which implies that $\mathcal{A}$ is diagonally dominant. By Theorem 3 of [24] it follows that $\mathcal{A}$ is positive semidefinite.

If $p>1$, then let $\lambda$ be an $\mathrm{H}$-eigenvalue of $\mathcal{A}$, and $\lambda<0$. By Theorem 5 we have $\lambda \in \Phi^{p}(\mathcal{A})$, which implies that

$$
\sum_{i \in[n]}\left[\frac{r_{i}^{p}(\mathcal{A})}{\left|\lambda-a_{i \cdots i}\right|}\right]^{\frac{p}{(m-1)(p-1)}} \geq 1 .
$$

However, it follows from $a_{i i \cdots i}>0$ for all $i \in[n]$ that

$$
\sum_{i \in[n]}\left[\frac{r_{i}^{p}(\mathcal{A})}{\left|a_{i \cdots i}\right|}\right]^{\frac{p}{(m-1)(p-1)}}>\sum_{i \in[n]}\left[\frac{r_{i}^{p}(\mathcal{A})}{\left|\lambda-a_{i \cdots i}\right|}\right]^{\frac{p}{(m-1)(p-1)}} \geq 1,
$$


which implies

$$
\left\|\delta_{p}(\mathcal{A})\right\|_{q}>1
$$

which contradicts $\left\|\delta_{p}(\mathcal{A})\right\|_{q} \leq 1$. This completes the proof.

Example 3 Let $\mathcal{A} \in \mathbb{R}^{[4,2]}$ be a symmetric tensor with elements defined as follows:

$$
a_{1111}=21, \quad a_{1222}=a_{2122}=a_{2212}=a_{2221}=-3, \quad a_{2222}=8,
$$

and the remaining $a_{i_{1} i_{2} i_{3} i_{4}}=0$. By computation,

$$
\left|a_{2222}\right|=8<9=r_{2}^{\Delta_{2}}(\mathcal{A})=\sum_{\substack{\left.i_{2}, i_{3}, i_{4}\right) \in \Delta_{2}, \delta_{2 i_{2} i_{3} i_{4}=0}}}\left|a_{2 i_{2} i_{3} i_{4}}\right|,
$$

which means that the statement (I) of Proposition 2.4 in [1] does not hold, and hence we cannot use Proposition 2.4 in [1] to determine the positive definiteness of $\mathcal{A}$. However, it is easy to verify that $\mathcal{A}$ is a $\infty$-norm $\mathrm{SDD}$ tensor. By Theorem $7, \mathcal{A}$ is positive definite.

\section{Conclusions}

In this paper, we proposed a new class of nonsingular tensors ( $p$-norm SDD tensors) and proved that the class of $p$-norm SDD tensors is a subclass of strong $\mathcal{H}$-tensors. Furthermore, we presented a new eigenvalue inclusion set, which is tighter than those provided by Li et al.[1] in some case. Based on this set, we presented a checkable sufficient condition for the positive (semi)definiteness of an even-order symmetric tensor.

\section{Competing interests}

The authors declare that they have no competing interests.

\section{Authors' contributions}

Both authors contributed equally to this work. Both authors read and approved the final manuscript.

\section{Acknowledgements}

This work was supported by the National Natural Science Foundations of China (11361074, 11501141), Natural Science Foundations of Yunnan Province (2013FD002) and Foundation of Guizhou Science and Technology Department ([2015]2073).

Received: 1 May 2016 Accepted: 23 June 2016 Published online: 08 July 2016

\section{References}

1. Li, C, Zhou, J, Li, Y: A new Brauer-type eigenvalue localization set for tensors. Linear Multilinear Algebra 64, 727-736 (2016)

2. Kofidis, E, Regalia, PA: On the best rank-1 approximation of higher-order supersymmetric tensors. SIAM J. Matrix Anal. Appl. 23, 863-884 (2002)

3. Qi, L: Eigenvalues of a real supersymmetric tensor. J. Symb. Comput. 40, 1302-1324 (2005)

4. Yang, Y, Yang, Q: Further results for Perron-Frobenius theorem for nonnegative tensors. SIAM J. Matrix Anal. Appl. 31 2517-2530 (2010)

5. Lim, LH: Singular values and eigenvalues of tensors: a variational approach. In: CAMSAP '05: Proceedings of the IEEE International Workshop on Computational Advances in Multi-Sensor Adaptive Processing, pp. 129-132 (2005)

6. Bose, N, Modaress, A: General procedure for multivariable polynomial positivity with control applications. IEEE Trans. Autom. Control 21, 596-601 (1976)

7. Cartwright, D, Sturmfels, B: The number of eigenvalues of a tensor. Linear Algebra Appl. 438, 942-952 (2013)

8. Chang, KC, Pearson, K, Zhang, T: Perron-Frobenius theorem for nonnegative tensors. Commun. Math. Sci. 6, 507-520 (2008)

9. Ding, W, Qi, L, Wei, Y: $\mathcal{M}$-Tensors and nonsingular $\mathcal{M}$-tensors. Linear Algebra Appl. 439, 3264-3278 (2013)

10. Hu, S, Huang, Z, Ling, C, Qi, L: On determinants and eigenvalue theory of tensors. J. Symb. Comput. 50, 508-531 (2013) 
11. Kolda, TG, Mayo, GR: Shifted power method for computing tensor eigenpairs. SIAM J. Matrix Anal. Appl. 32 1095-1124 (2011)

12. Kay, DC: Theory and Problems of Tensor Calculus. McGraw-Hill, New York (1988)

13. Qi, L: Eigenvalues and invariants of tensors. J. Math. Anal. Appl. 325, 1363-1377 (2007)

14. Yang, Q, Yang, Y: Further results for Perron-Frobenius theorem for nonnegative tensors II. SIAM J. Matrix Anal. Appl. 32, 1236-1250 (2011)

15. Zhang, T, Golub, GH: Rank-1 approximation of higher-order tensors. SIAM J. Matrix Anal. Appl. 23, 534-550 (2001)

16. Zhang, L, Qi, L, Zhou, G: M-Tensors and some applications. SIAM J. Matrix Anal. Appl. 35, 437-542 (2014)

17. Li, C, Li, Y, Kong, X: New eigenvalue inclusion sets for tensors. Numer. Linear Algebra Appl. 21, 39-50 (2014)

18. Brauer, A: Limits for the characteristic roots of a matrix II. Duke Math. J. 14, 21-26 (1974)

19. Li, C, Li, Y: An eigenvalue localization set for tensors with applications to determine the positive (semi-)definiteness of tensors. Linear Multilinear Algebra 64, 587-601 (2016)

20. Kostić, V: On general principles of eigenvalue localizations via diagonal dominance. Adv. Comput. Math. 41, 55-75 (2015)

21. Li, C, Wang, F, Zhao, J, Zhu, Y, Li, Y: Criterions for the positive definiteness of real supersymmetric tensors. J. Comput. Appl. Math. 255, 1-14 (2014)

22. Shao, J: A general product of tensors with applications. Linear Algebra Appl. 439, 2350-2366 (2013)

23. Kannan, MR, Shaked-Monderer, N, Berman, A: Some properties of strong $\mathcal{H}$-tensors and general $\mathcal{H}$-tensors. Linear Algebra Appl. 476, 42-55 (2015)

24. Qi, L, Song, Y: An even order symmetric B tensor is positive definite. Linear Algebra Appl. 457, 303-312 (2014)

\section{Submit your manuscript to a SpringerOpen ${ }^{\circ}$ journal and benefit from:}

- Convenient online submission

Rigorous peer review

- Immediate publication on acceptance

- Open access: articles freely available online

- High visibility within the field

- Retaining the copyright to your article 